\title{
Magnetic Properties and Structure of Non-Oriented Electrical Steel Sheets after Different Shape Processing
}

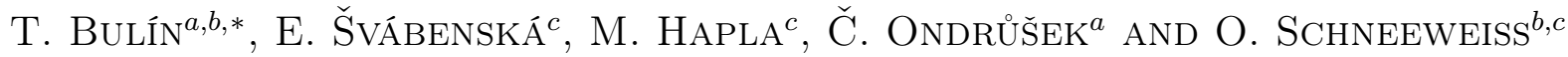 \\ ${ }^{a}$ Brno University of Technology, Faculty of Electrical Engineering and Communication, \\ Technická 3058/10, 61600 Brno, Czech Republic \\ ${ }^{b}$ CEITEC IPM, Institute of Physics of Materials ASCR, Žižkova 22, 61662 Brno, Czech Republic \\ ${ }^{c}$ Institute of Physics of Materials ASCR, Žižkova 22, 61662 Brno, Czech Republic
}

\begin{abstract}
Non-oriented electrical steel sheets are the most often used materials in electrical rotary machines. Raw sheets must be formatted by various methods, e.g., punching, laser, spark or water stream cutting. Basic parameters of magnetic, electrical, and mechanical properties of the sheets are usually obtained from the producer, but namely magnetic properties are changed in dependence on additional machining processes. The aim of this study is to describe changes in magnetic properties after punching, laser or spark cutting of the original sheets M470-50A. The basic information about the structure of the cut edges of samples was obtained by optical microscopy and scanning electron microscopy. For the magnetic measurements toroidal samples were prepared by the mentioned technologies. The magnetic parameters were yielded from the measuring of magnetic hysteresis loops in dependence on saturation fields. The results are discussed from the point of view of applied cutting methods with the aim to obtain the best magnetic parameters and consequently determining the depth of the influenced material. After laser cutting, important decrease in remanence and increase in total losses were observed in comparison with the punched samples.
\end{abstract}

DOI: 10.12693/APhysPolA.131.819

PACS/topics: 75.60.Ej, 75.20.En

\section{Introduction}

Non-oriented Si steel sheets are the priority material for magnetic circuits in electrical rotary machines. Their machining influences magnetic properties. The amount of material used for construction is changing in dependence on requested parameters of a machine and on final properties of the component made from the sheets [1]. The producers of the sheets usually measure magnetic parameters using the Epstein frame and the sheets are cut by guillotine. Electrical machines are generally made from the sheets that are produced by punching, but sometimes laser cutting is used for prototype or very small series of machines. Each of this type of processing influences magnetic properties of material [2-4].

The goal of this paper is to describe changes in parameters of magnetic properties due to application of different cutting techniques for toroid shaped samples and determining the depth of degradation. The main attention will be given to the deterioration and consequently improvement of permeability and total core losses after removing thin influenced layer of material.

\section{Experimental}

The investigated samples were produced from commercially non-oriented Si steel M470-50A with thickness of

*corresponding author; e-mail: xbulin01@stud.feec.vutbr.cz
$0.5 \mathrm{~mm}$ [5]. This steel is composed of iron with $1.7 \mathrm{wt} \%$ silicon. The samples in shape of rings were prepared by punching, laser, and spark erosion cutting. The punching of the rings was carried out using NK 8 from Müller Weingarten for punching stator and rotor steel sheets. Laser cutting was done by Byspeed 3015 .

The basic information of structure was obtained by optical microscopy $(\mathrm{OM})$ and scanning electron microscopy (SEM). The samples were etched by $2 \%$ Nital solution to make grains/grain boundaries visible.

The magnetic parameters were acquired from the measurement of magnetic hysteresis loops in dependence on saturation fields and frequencies. Equipment Remagraph-Remacomp C-710 (Magnet-Physik Dr. Steingroever $\mathrm{GmbH}$ ) was used with the ring core samples for AC measurements and quasistatic measurements of saturation magnetization and hysteresis losses. This device is measuring the magnetic flux through the coil based on the principle of the Faraday law of electromagnetic induction. Outer diameter of the first type of samples was $120 \mathrm{~mm}$, inner diameter was $105 \mathrm{~mm}$, height of this sample was $7.5 \mathrm{~mm}$ and weight was $158 \mathrm{~g}$. Number of turns of primary winding was 243 and secondary winding had 50 turns. The second type of samples had outer radius $80 \mathrm{~mm}$ and inner radius $70 \mathrm{~mm}$, height of this samples was $5 \mathrm{~mm}$ and weight was $45.5 \mathrm{~g}$. Number of turns of primary winding was 162 and secondary winding had 40 turns. The properties were measured in frequency range $0-2000 \mathrm{~Hz}$ at room temperature. Remagraph was used for the quasistatic measurements and Remacomp was used for the other measurements. Larger samples were created by punching or laser cutting while smaller 
samples were created by spark cutting. The aspect ratio of these samples was 1.14 and it is close to ideal ratio for toroid sample which should be less than 1.1. The main advantage of this measurement is proximity of influenced edges in tested toroid samples.

After the measurements of prepared laser and spark cut samples additional processing was applied. The thin layers of material close to the cutting edges (approximately $0.2 \mathrm{~mm}$ ) were finely ground off. In the case of spark cut samples thin layers at the edges were removed by sandpaper. These modified samples were then measured again.

\section{Results}

The basic information on structure from OM is shown in Figs. 1 and 2. Changes in grain sizes were not observed in any sample. An effect of plastic deformation occurring near the cut edge on the punched sample can be seen in Fig. 1. The results from SEM confirmed OM results.

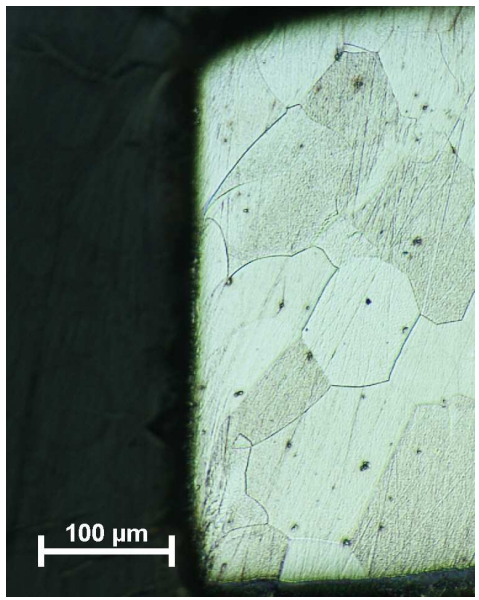

Fig. 1. OM sheet, punching, etching, $200 \times$ magnification.

Magnetic measurement proved that the heat stress can make significant changes in the material and its properties. The most noticeable change compared to the usual shape of hysteresis curve can be observed on laser cut sample. It looks like composition of two curves. The slope of curves for small excitation is greater than for higher excitation. The comparison of the hysteresis curves for different processing and excitation is shown in Fig. 3. The main difference can be seen in value of remanence $B_{r}$ which is significantly lower for the laser cut sample than for the rest of the samples.

After removing the edges on the laser cut samples a significant improvement of magnetic properties was detected (Fig. 4). Hysteresis curve of adjusted laser cut sample (Laser1) is almost the same as the curve of the sample after spark cutting. The spark cut and edge ground sample (Spark1) showed also an insignificant improvement.

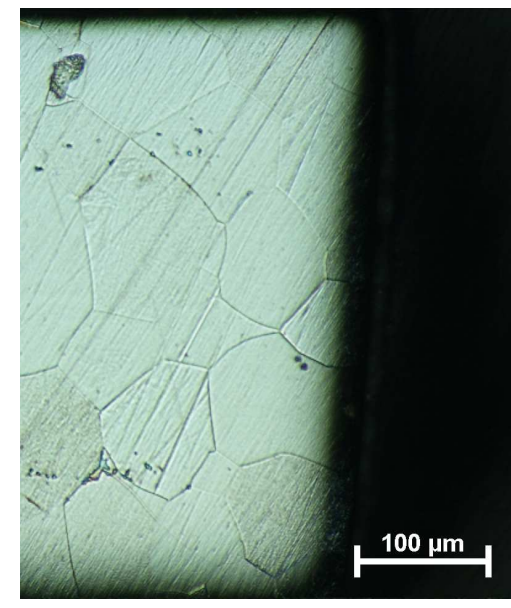

Fig. 2. OM sheet, laser cutting, etching, $200 \times$ magnification.



Fig. 3. Hysteresis curves for different processing at $1.5 \mathrm{~T}$ and $50 \mathrm{~Hz}$.

The changes in magnetic parameters can be more clearly seen on value of permeability (Fig. 5) because this is very sensitive parameter on various structure parameters. Permeability of laser cut sample almost does not increase while the rest of the samples or the edge ground laser cut sample shows substantial increase for small magnetic flux density.

Comparison of total magnetic losses for all samples shows their exponential increase with increasing induction. The exception is laser cut sample where this dependence is almost linear for the small magnetic flux density (Fig. 6).

At higher values of excitation value magnetic losses and permeability become the same for all compared samples. Laser cut sample has higher magnetic losses for lower magnetic flux density due to structural changes in the surface layers of edges and consequently deterioration of conditions for the movement of domain walls.

DC measurements of magnetic properties proved previous observed changes. Curve of initial magnetization has the lowest slope for laser cut sample. These differences (Fig. 7) can be observed for the toroid samples 


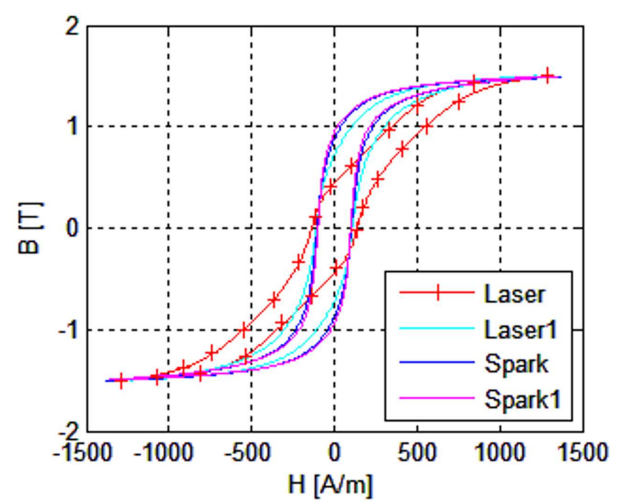

Fig. 4. Comparison hysteresis curves after removing cutting edges.

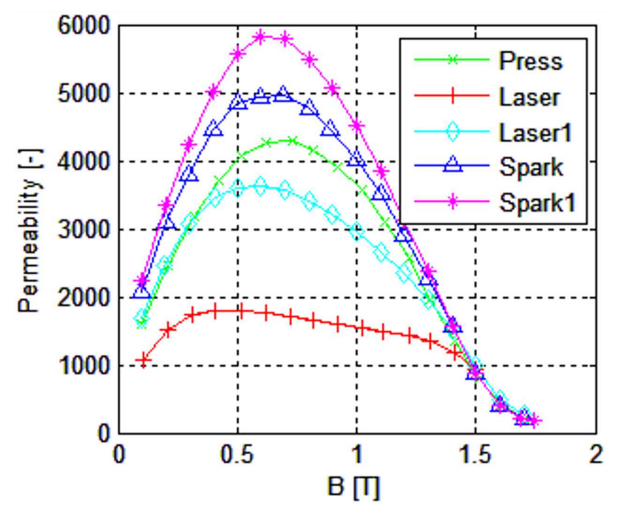

Fig. 5. Dependence of permeability on magnetic flux density at $50 \mathrm{~Hz}$.

only. By measuring at different frequencies it was found that permeability of sample after laser cutting is already deteriorated and with increasing frequency is not so significant drop as at other samples.

Spark cutting is one of the gentlest processing methods and therefore the effect of material damage is minimal

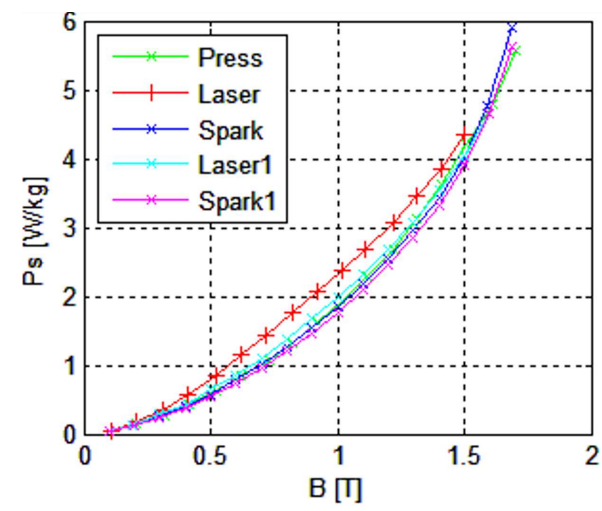

Fig. 6. Dependence of total magnetic losses Ps on magnetic flux density $B$ at $50 \mathrm{~Hz}$.

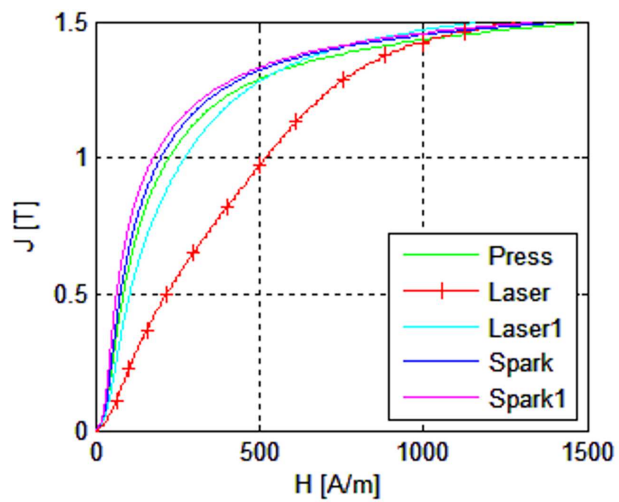

Fig. 7. The quasistatic magnetization curves measured by Remagraph.

at the edges. The smaller size was used for comparing magnetic properties of the rest of material after punching and laser cutting. Smaller area of the ferromagnetic does not influence results too much and measured curves show even better magnetic properties.

\section{Conclusions}

Obtained results show that magnetic parameters of electrical steel sheets M470-50A are influenced by processing. The sample after spark cutting exhibits the best parameters. The shape of the hysteresis curve after laser cutting was deformed and permeability is extremely deteriorated for small magnetic flux density. Improvement of magnetic parameters was reached after removing surface layer of influenced edges. The total losses decrease and permeability increases for small magnetic flux density.

\section{Acknowledgments}

This research work has been carried out in the Centre for Research and Utilization of Renewable Energy (CVVOZE). Authors gratefully acknowledge financial support from the Ministry of Education, Youth and Sports of the Czech Republic under NPU I programme (project No. LO1210). They also acknowledge partner collaboration in frame of project of Technology Agency of the Czech Republic No. TE02000232.

\section{References}

[1] Y. Kurosaki, H. Mogi, H. Fujii, T. Kubota, M. Shiozaki, J. Magn. Magn. Mater. 320, 2474 (2008).

[2] E.G. Araujo, J. Schneider, K. Verbeken, G. Pasquarella, Y. Houbaert, IEEE Trans. Magn. 46, 213 (2010).

[3] R. Siebert, J. Schneider, E. Beyer, IEEE Trans. Magn. 50, 1 (2014).

[4] W. Shi, J. Liu, C. Li, J. Wuhan Univ. Technol. Sci. Ed. 29, 1246 (2014).

[5] Non-Oriented Electrical Steels, Acroni d.o.o. 